\title{
Iniquidade social e câncer de mama feminino: análise da mortalidade
}

\section{Social iniquity and breast cancer in women: mortality analysis}

\author{
Daniela de Almeida Pereira Duarte ${ }^{1}$, Mário Círio Nogueira² (1), \\ Maria da Consolação Magalhães ${ }^{3}$ (1), Maria Teresa Bustamante-Teixeira ${ }^{4}$ (1) \\ 'Departamento de Saúde Coletiva, Faculdade de Medicina, Universidade Federal de Juiz de Fora (UFJF), Juiz de Fora, MG, Brasil \\ 2Departamento de Saúde Coletiva, Faculdade de Medicina, Universidade Federal de Juiz de Fora (UFJF), Juiz de Fora, MG, Brasil. \\ ${ }^{3}$ Hospital Universitário, Universidade Federal de Juiz de Fora (UFJF), Juiz de Fora, MG, Brasil. \\ ${ }^{4}$ Departamento de Saúde Coletiva, Faculdade de Medicina, Universidade Federal de Juiz de Fora (UFJF), Juiz de Fora, MG, Brasil.
}

Como citar: Duarte DAP, Nogueira MC, Magalhães MC, Bustamante-Teixeira MT. Iniquidade social e câncer de mama feminino: análise da mortalidade. Cad Saúde Colet, 2020;28(4):465-476. https://doi.org/10.1590/1414-462X202028040360

\section{Resumo}

Introdução: Apesar das ações para prevenção, o câncer de mama (CAM) no Brasil apresenta elevada mortalidade, provavelmente devido à identificação do tumor em estádios avançados. Objetivo: Analisar a mortalidade por CAM nas microrregiões de saúde de Minas Gerais (MG), de 2013 a 2017 e sua possível associação com a desigualdade social. Método: Estudo ecológico, cuja unidade de análise foram as microrregiões de saúde de MG. Dados de mortalidade, sociodemográficos e de saúde foram extraídos do SIM, IBGE, PROADESS e DATASUS. Foram calculadas taxas de mortalidade específicas e padronizadas por idade, construídos mapas temáticos e realizadas análises estatísticas utilizando o Índice de Moran e a regressão simples e múltipla. Resultados: De 2013 a 2017, ocorreram em MG 7.571 óbitos por CAM. As microrregiões com maior mortalidade estão localizadas no Centro e Leste e, com menor, no Norte e Nordeste. A maioria das variáveis apresentaram alto coeficiente de variação e foram significativas no modelo de regressão linear simples. Nos modelos múltiplos distal e proximais, somente o grau de urbanização foi significativa. Todas as variáveis apresentaram autocorrelação espacial significativa e dependência espacial. Conclusão: Altas taxas de mortalidade nas microrregiões mais urbanizadas podem ser explicadas por fatores reprodutivos, comportamentais e distribuição dos recursos de saúde, presentes nos grandes centros urbanos.

Palavras-chave: neoplasia da mama; iniquidade social; estudo ecológico.

\begin{abstract}
Introduction: Despite the preventive actions, breast cancer (BC) in Brazil has a high mortality, probably due to the identification of the tumor in advanced stages. Objective: To analyze mortality from $B C$ in the health micro-regions of Minas Gerais (MG), 2013-2017, and its possible association with social inequality. Method: Ecological study, whose unit of analysis was the health micro-regions of MG. Mortality, sociodemographic and health data were extracted from SIM, IBGE, PROADESS, and DATASUS. Specific and age-standardized mortality rates were calculated, thematic maps were constructed, and statistical analyzes were performed using the Moran Index and multiple simple regression. Results: From 2013-2017 there were 7,571 deaths from BC in MG. The deadliest microregions are in the Center and East; the smallest in the North and Northeast. Most variables had a high coefficient of variation and were significant in the simple linear regression model. In the multiple distal and proximal models, only the degree of urbanization was significant. All variables showed significant spatial autocorrelation and spatial dependence. Conclusion: High mortality rates in the most urbanized micro-regions can be explained by reproductive, behavioral factors and the distribution of health resources, present in large urban centers.
\end{abstract}

Keywords: breast neoplasia; social inequity; ecological study.

Trabalho realizado na Universidade Federal de Juiz de Fora (UFJF) - Juiz de Fora (MG), Brasil.

Correspondência: Daniela de Almeida Pereira. E-mail: danalmeidap@yahoo.com.br

Conflito de interesses: nada a declarar.

Recebido em: Dez. 01, 2016. Aprovado em: Nov. 21, 2019
Este é um artigo publicado em acesso aberto (Open Access) sob a licença Creative Common Attribution, que permite uso, qualquer meio, sem restrições desde que o trabalho original seja corretamente citado. 


\section{INTRODUÇÃO}

O crescimento demográfico, associado ao envelhecimento populacional e ao desenvolvimento socioeconômico, contribui gradativamente com o aumento da incidência e mortalidade por câncer, tornando-o um grave problema de saúde pública. O câncer de mama (CAM) é o mais frequente no sexo feminino em 154 países e figura como a principal causa de mortalidade em 103 países'. A incidência e a mortalidade por CAM têm decrescido nos países desenvolvidos, porém situação inversa ocorre naqueles em desenvolvimento, devido a estilos de vida e comportamentos não saudáveis, dificuldade de acesso ao diagnóstico/tratamento ${ }^{2,3}$.

Mundialmente, o CAM representa a quinta causa de morte por câncer em geral, com taxa variando entre 2,7 e 36,9/100.000 mulheres'. No sexo feminino, no ano de 2016, o câncer de mama foi a principal causa de morte, com taxa padronizada de 14,6/100.000 mulheres ${ }^{4}$. Países da Ásia, Espanha, Noruega e Albânia apresentam as menores taxas de incidência e mortalidade, enquanto as maiores taxas são encontradas em Montenegro, Sérvia e República da Moldávia'.

No Brasil, observou-se tendência crescente da mortalidade por CAM a partir de 1980 e esta acompanhou o aumento dos indicadores positivos de nível socioeconômico; ressalta-se diminuição somente para as mulheres residentes nas capitais, desde o final da década de $90^{5}$. Em 2018, a taxa de mortalidade padronizada pela população mundial foi de13,22 óbitos/100.000 mulheres, sendo que as regiões Sul e Sudeste foram as que apresentaram as maiores taxas (14,14 e 14,10 por 100.000 mulheres, em 2017, respectivamente) $)^{6}$.

As desigualdades regionais na mortalidade por câncer de mama são, em parte, atribuíveis às variações nos fatores socioeconômicos e na disponibilidade e acessibilidade dos serviços de detecção e tratamento precoce ${ }^{2,3}$.

No Brasil, há grande desigualdade de renda: enquanto o $1 \%$ mais rico da população concentra cerca de $1 / 4$ da renda, a metade mais pobre não chega a acumular $10 \%$ de toda a renda do país ${ }^{7}$. Portanto, é relevante a realização de estudos que avaliem as implicações dessa má distribuição de renda na efetividade das políticas públicas de saúde. Desigualdades na mortalidade por CAM entre as regiões brasileiras estão associadas tanto ao índice de desenvolvimento humano quanto ao índice de exclusão ${ }^{8}$.

A adoção de medidas preventivas deve considerar não somente fatores biológicos, mas também os socioeconômicos e assistenciais de cada região para se estabelecer estratégias eficazes no rastreamento, diagnóstico precoce e tratamento, atendendo de forma específica e eficiente às demandas de cada área.

O objetivo deste estudo foi analisar a mortalidade por CAM no estado de Minas Gerais (MG) e suas microrregiões de saúde e sua possível associação com a desigualdade social.

\section{MÉTODO}

Estudo quantitativo, do tipo observacional, cujo método de investigação caracteriza-se por um estudo epidemiológico ecológico, que tomou como unidade de análise as microrregiões de saúde de Minas Gerais.

Minas Gerais situa-se na região sudeste do Brasil, é o quarto estado em extensão territorial com superfície de 586.522,122 km², possui 853 municípios, 13 macrorregiões e 77 microrregiões de saúde. Sua população em 2010 era de 19.597 .330 habitantes, destes, 9.955 .453 são mulheres ${ }^{9}$.

Foram analisados dados de mortalidade do período de 2013 a 2017 e dados sociais, demográficos e de saúde referentes ao ano de 2010, extraídos das seguintes fontes:

- $\quad$ Sistema de Informação Sobre Mortalidade(SIM): total de óbitos, óbitos por CAM (C50 da CID - 10a revisão), causas externas e causas mal definidas (capítulos XX e XVIII), extraídos por faixa etária (intervalos de cinco anos) e microrregião de residência;

- Departamento de Informática do SUS (DATASUS): cobertura de equipes da saúde da família, razão de mamografia, nascidos vivos, número de médicos, número de enfermeiros e população estimada para o ano de 2015 pela Rede Interagencial de Informações para a Saúde (RIPSA); 
- Instituto Brasileiro de Geografia e Estatística (IBGE): população rural e urbana e variáveis do Índice de Vulnerabilidade na Saúde (IVS). Informações indisponíveis foram solicitadas via sítio eletrônico para acesso à informação do governo federal ${ }^{10}$;

- Programa de Avaliação do Desempenho do Sistema de Saúde (PROADESS): Gasto Público em Saúde per Capita, Índice de Desenvolvimento Humano Regional (IDHR), Número de Mamógrafos/Habitantes, Distância Percorrida para Realização de Mamografia (DPRM) e Leitos/1.000 Habitantes.

Os óbitos por causas mal definidas foram redistribuídos obedecendo os seguintes passos (Equações 1 e 2):

1) Cálculo do Peso de causas mal definidas (PCMD) dentro do número total de óbitos:

$P C M D=\frac{(\text { Total de Óbitos Femininos }- \text { Óbitos Causas Externas })}{(\text { Total de Óbitos Femininos -Óbitos Causas Externas })-(\text { Óbitos Causas mal definidas })}$

2) Cálculo do fator de correção (FC):

$$
F C=1+\frac{(P C M D-1)}{2}
$$

Em seguida, multiplicou-se o fator de correção pelo número de óbitos por CAM em cada faixa etária ( 5 anos). Foram estimadas taxas médias anuais de mortalidade para o período de 2013 a 2017, padronizadas pela população mundial ${ }^{11}$. O cálculo da taxa de mortalidade, do grau de urbanização e do número de médicos e enfermeiros foram baseados no livro de Indicadores Básicos para Saúde no Brasil' ${ }^{12}$.

O IVS é composto por variáveis divididas em duas dimensões: Saneamento e Socioeconômica e foi calculado conforme a proposta de Pitchon et al. ${ }^{13}$.

A análise exploratória dos dados compreendeu: construção de mapas temáticos, teste da autocorrelação espacial (Índice de Moran Global e Local - LISA) e regressão linear. As proporções de cada indicador foram classificadas em quintis, expressos nos mapas segundo escala com tons mais claros para os menores valores e mais escuros para os maiores valores.

A prioridade de inserção das variáveis no modelo de regressão baseou-se na proposta de Victora et al. ${ }^{14}$, cuja inclusão de elementos num modelo múltiplo deve fundamentar-se num quadro conceitual que descreve hierarquicamente a relação entre os fatores de risco.

Diferenças nas taxas de incidência e mortalidade por câncer de mama nos países e suas regiões podem ser explicadas não só por fatores biológicos, ambientais, comportamentais e sociais, mas também pelas estratégias governamentais adotadas para prevenção, detecção e diagnóstico precoces ${ }^{15}$.

Com base em evidências científicas, nas recomendações nacionais e internacionais, cada país, de acordo com seu grau de desenvolvimento, condições econômicas, territoriais, socioeconômicas e estruturais, define sua política para o controle do câncer. Barreiras socioeconômicas e culturais impedem que as mulheres busquem atendimento para o câncer de mama em momento oportuno e fatores como disparidades no acesso aos serviços de saúde, baixa cobertura, falta de pessoal e infraestrutura e financiamento adequados atrasam ainda mais o diagnóstico ${ }^{16}$. Dessa maneira, fatores socioeconômicos devem condicionar a configuração e oferta de estratégias específicas e adequadas para cada região/município, pois a disponibilidade e qualidade dos serviços de saúde impactam diretamente a sobrevida dos pacientes.

Baseado nestes autores, foi construído um modelo conceitual hierarquizado, tendo como variável dependente a taxa de mortalidade por CAM e variáveis independentes organizadas em dois blocos, um distal composto pelas variáveis IDH, IVS, renda, grau de urbanização e população feminina. No bloco proximal, foram incluídas as variáveis gasto público per capita em saúde, médicos, enfermeiros, leitos, número de mamógrafos, cobertura ESF, distância percorrida para realização de mamografia e razão de mamografia.

Foi feita a regressão linear simples para cada variável distal e proximal. Todas as variáveis do bloco distal significativas nos modelos simples foram incluídas no modelo múltiplo do 
bloco distal. Para avaliar associação das variáveis do bloco proximal, as variáveis do bloco distal que tiveram associação no modelo múltiplo $(p \leq 0,20)$ foram incluídas para controle de confundimento. Posteriormente foram inseridas no modelo cada uma das variáveis proximais, sendo consideradas significativas aquelas que mostraram valor de $p<0,05$. Os resíduos de todos os modelos de regressão linear foram testados para a normalidade e homocedasticidade de sua distribuição (respectivamente com os testes de Jarque-Bera e Breusch-Pagan) e também foram testados para autocorrelação espacial com o teste de Moran.

A extração, processamento, geoprocessamento e análise dos dados foram realizados pelos programas TABWIN versão 4.1.5, Microsoft Excel 2007, TerraView versão 4.2.2 e GeoDa versão 1.12.1.161.

A coleta das informações foi realizada em bases de dados secundários e não identificados, portanto foi solicitada, ao Comitê de Ética da Universidade Federal de Juiz de Fora, dispensa do Termo de Consentimento Livre e Esclarecido, aprovado sob o parecer nº 1376660.

\section{RESULTADOS}

Entre 2013 e 2017, ocorreram em MG 7.571 óbitos por CAM, com uma taxa de mortalidade média anual padronizada por idade de 11,8/100.000 mulheres. As cinco microrregiões que apresentaram em ordem decrescente as maiores taxas foram: Curvelo (16,8); Coronel Fabriciano/ Timóteo (15,5); Contagem (15,2); Belo Horizonte/Nova Lima/Caeté (15,1); e Ouro Preto $(15,0)$. As menores taxas foram encontradas, em ordem crescente, em Coração de Jesus $(1,8)$; Itaobim $(4,0)$; e Pedra Azul $(4,5)$ (Figura 1).

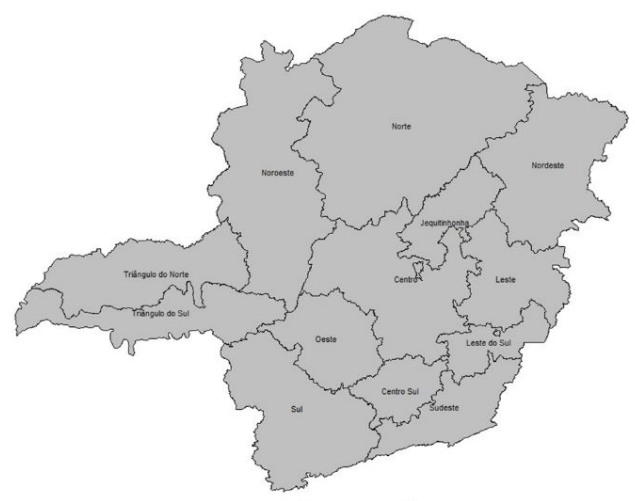

MACRORREGIÕES DE SAÚDE - MG

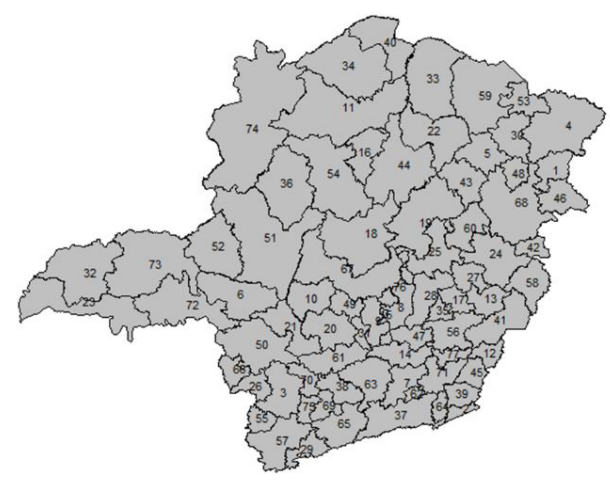

MICRORREGIÕES DE SAÚDE - MG

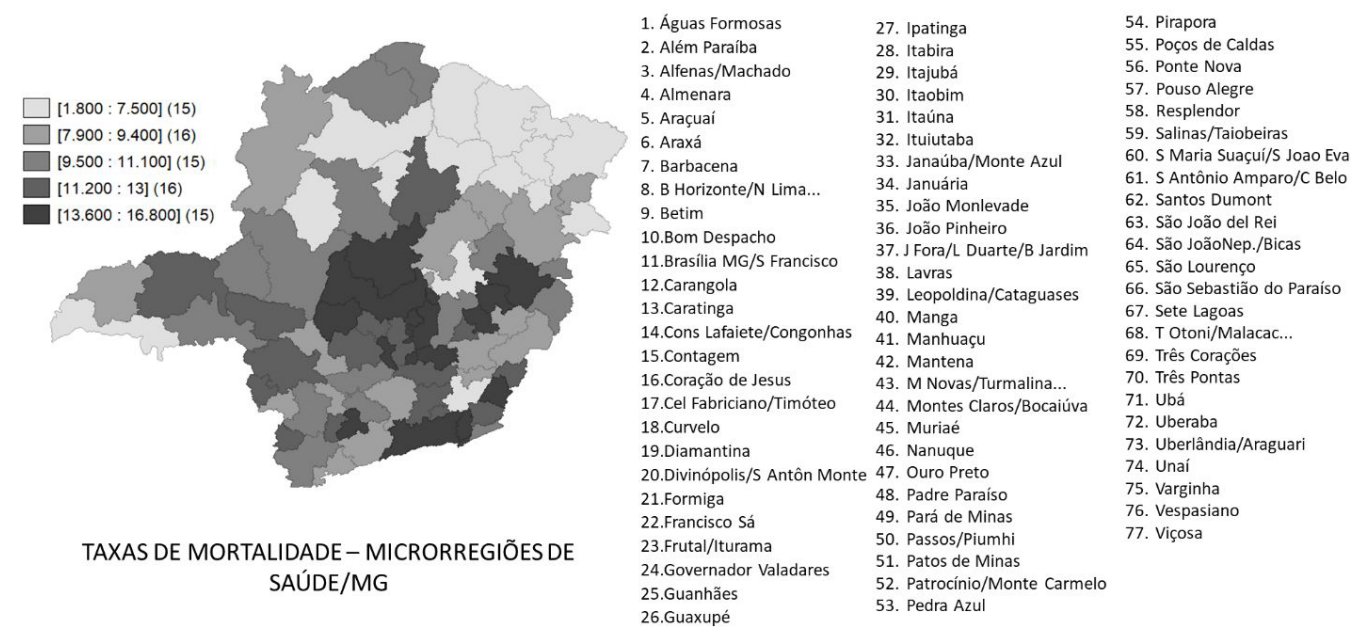

Figura 1. Macrorregiões e Microrregiões de Minas Gerais e Taxas de Mortalidade por Câncer de Mama Padronizadas pela População Mundial - Regiões de Saúde de Minas Gerais (2013-2017) 
Os indicadores socioeconômicos e de saúde apresentam alto coeficiente de variação (>20\%), exceto IDH e grau de urbanização (Tabela 1). Todas as variáveis tiveram autocorrelação espacial significativa.

Tabela 1. Taxas de mortalidade por câncer de mama* e indicadores socioeconômicos e de saúde** das mulheres residentes em Minas Gerais, Brasil

\begin{tabular}{|c|c|c|c|c|c|c|c|c|}
\hline Variável (n=77) & Média & $\begin{array}{l}\text { Desvio } \\
\text { Padrão }\end{array}$ & $\begin{array}{c}\text { Valor } \\
\text { Mínimo }\end{array}$ & Q1 & Médiana & Q3 & $\begin{array}{c}\text { Valor } \\
\text { Máximo }\end{array}$ & $\begin{array}{c}\text { Coeficiente } \\
\text { de Variação } \\
(\%)\end{array}$ \\
\hline Taxa de Mortalidade & 10,27 & 3,05 & 1,80 & 8,40 & 10,40 & 12,40 & 16,80 & 29,72 \\
\hline IDH & 0,69 & 0,04 & 0,56 & 0,65 & 0,71 & 0,73 & 0,79 & 7,02 \\
\hline IVS & 0,38 & 0,21 & 0,14 & 0,22 & 0,29 & 0,5 & 0,9 & 56,11 \\
\hline Grau de Urbanização (\%) & 78,84 & 13,43 & 42,46 & 70,40 & 83,28 & 89,49 & 99,66 & 17,04 \\
\hline População Feminina & 129.292 & 195.935 & 23.033 & 57.527 & 88.521 & 135.496 & 1.675 .279 & 151.545 \\
\hline Renda & 580,83 & 184,76 & 246,33 & 442,80 & 610,20 & 695,30 & $1.248,13$ & 31,80 \\
\hline Gastos per capita em saúde & 365,68 & 78,74 & 253,50 & 305,20 & 349,10 & 412,20 & 555,20 & 21,53 \\
\hline Médicos & 1,09 & 0,58 & 0,32 & 0,74 & 0,97 & 1,29 & 3,72 & 53,42 \\
\hline Enfermeiros & 0,60 & 0,12 & 0,38 & 0,52 & 0,58 & 0,67 & 1,04 & 20,67 \\
\hline Leitos Hospitalares & 2,00 & 0,75 & 0,70 & 1,40 & 2,00 & 2,50 & 4,30 & 37,82 \\
\hline Número de Mamógrafos & 2,04 & 1,07 & 0,00 & 1,40 & 2,20 & 2,70 & 5,30 & 52,88 \\
\hline Cobertura ESF (\%) & 70,52 & 15,34 & 32,35 & 58,35 & 70,16 & 80,91 & 100,00 & 21,76 \\
\hline Distância Mamografia & 39,25 & 47,32 & 2,10 & 11,40 & 22,40 & 42,70 & 270,90 & 120,57 \\
\hline Razão Mamografia & 0,27 & 0,12 & 0,00 & 0,19 & 2,80 & 0,36 & 0,60 & 46,17 \\
\hline
\end{tabular}

Fonte: SIM/Datasus/MS ${ }^{17}$, Censo Demográfico 20109 , PROADESS 201018; ESF: Estratégia Saúde da Família; N= 77 corresponde ao número de microrregiões de saúde de Minas Gerais; *Taxas do período de 2013 a 2017 por 100.000 habitantes; **Indicadores socioeconômicos e de saúde do ano de 2010

Observa-se alta vulnerabilidade na saúde nas microrregiões localizadas nas macrorregiões Norte, Noroeste, Nordeste, Jequitinhonha e Leste, enquanto que no Triângulo, Centro e Sul constatou-se menor vulnerabilidade. A vulnerabilidade em saúde apresentou alta correlação negativa com grau de urbanização, IDH e renda per capita (Figura 2).

No modelo de regressão linear simples, todas as variáveis foram significativas, com exceção de razão de enfermeiros e de leitos hospitalares. No bloco socioeconômico/demográfico, as variáveis significativas com maiores coeficientes de determinação $\left(R^{2}\right)$ foram: grau de urbanização $(42,7 \%)$, IDH $(38,7 \%)$, renda média per capita $(35,8 \%)$ e IVS $(35,0 \%)$. No bloco recursos/acesso, foi razão de médicos (por 1.000 habitantes) (Tabela 2).

No modelo múltiplo distal, a única variável significativa foi grau de urbanização. Ao incluir nos modelos múltiplos as variáveis proximais, optou-se por manter a variável população feminina juntamente com grau de urbanização para controle de confundimento. A única variável que permaneceu significativa foi grau de urbanização (Tabela 3) .

\section{DISCUSSÃO}

As 77 microrregiões de saúde de Minas Gerais apresentaram taxas de mortalidade médias anuais por câncer de mama que variaram entre 1,8 a 16,8/100.000 mulheres. Das cinco microrregiões de saúde com maior mortalidade, quatro estão localizadas na macrorregião Centro, uma das regiões mais desenvolvidas e urbanizadas do estado. A quinta, Coronel Fabriciano/Timóteo, pertence à macrorregião Leste, porém faz fronteira com a macrorregião 

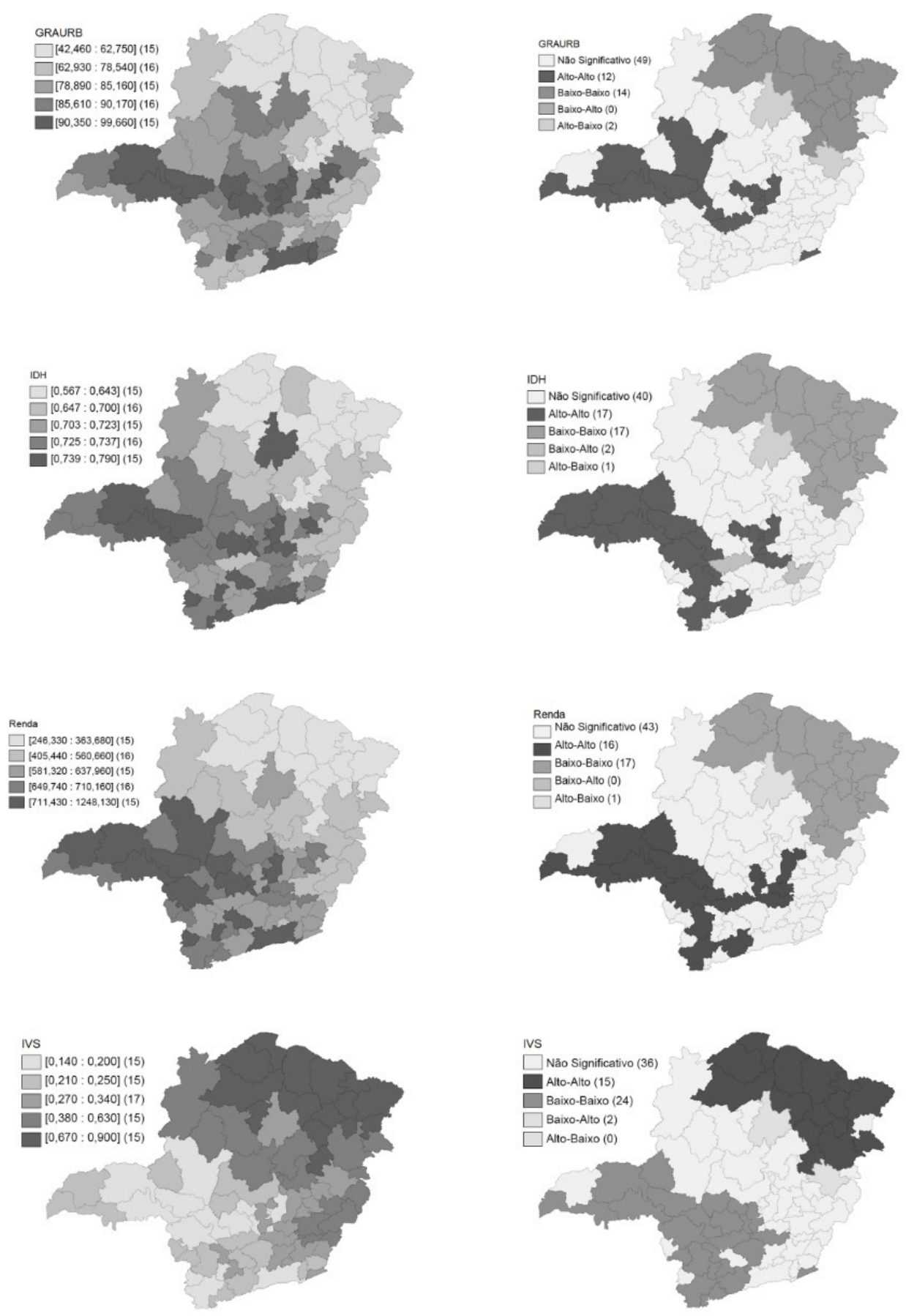

Figura 2. Grau de Urbanização (GRAURB), Índice de Desenvolvimento Humano (IDH), Renda, Índice de Vulnerabilidade na Saúde (IVS) - Regiões de Saúde de Minas Gerais (2013-2017).

Centro. As microrregiões com menor mortalidade localizam-se nas macros Norte e Nordeste, menos urbanizadas.

Minas Gerais possui características geográficas, estruturais e econômicas muito parecidas com o Brasil: regiões mais pobres e menos desenvolvidas ao Norte e Nordeste e mais ricas e desenvolvidas ao Centro e Sul. Isto demonstra a relevância das pesquisas realizadas no estado, pois podem retratar importantes situações sociais, econômicas e de saúde que ocorrem em 
Tabela 2. Modelos de Regressão Linear Simples

\begin{tabular}{lccc}
\multicolumn{1}{c}{ Variável (n=77) } & $\mathbf{R}^{\mathbf{2}}$ ajustado & Coeficiente & p-valor \\
\hline IDH & 0.387 & 0.391 & $<0.001$ \\
\hline IVS & 0.350 & -0.085 & $<0.001$ \\
\hline Grau de Urbanização (\%) & 0.427 & 0.149 & $<0.001$ \\
\hline População Feminina (log) & 0.188 & 1.802 & $<0.001$ \\
\hline Renda Média per capita & 0.358 & 0.010 & 0.002 \\
\hline Gastos per capita em saúde (R\$) & 0.106 & 0.013 & $<0.001$ \\
\hline Razão de Médicos* & 0.222 & 2.512 & 0.342 \\
\hline Razão de Enfermeiros* & -0.001 & 2.697 & 0.868 \\
\hline Leitos Hospitalares* & -0.012 & -0.077 & 0.001 \\
\hline Número de Mamógrafos** & 0.108 & 0.980 & $<0.001$ \\
\hline Cobertura ESF (\%) & 0.134 & -0.075 & 0.001 \\
\hline DPRM (Km) & 0.119 & -0.023 & 0.009 \\
\hline Razão Mamografia & 0.073 & 7.111 & 0.001 \\
\hline
\end{tabular}

*por 1.000 habitantes; **por 100.000 habitantes; ESF: Estratégia Saúde da Família; DPRM: Distância percorrida para realização de mamografia; $\mathrm{N}=77$ corresponde ao número de microrregiões de saúde de Minas Gerais

todo o país, apontando para onde devem ser direcionados os estudos mais abrangentes da realidade brasileira ${ }^{19}$.

No Brasil, as desigualdades regionais na mortalidade por câncer de mama persistem, embora tenha havido um decréscimo no período de 1980 a 2016. Tal como no presente estudo, as maiores taxas são observadas nas regiões mais desenvolvidas e urbanizadas do país ${ }^{8}$.

A microrregião de saúde que compreende a capital do estado, Belo Horizonte/Nova Lima/ Caeté, foi a quarta microrregião com maior mortalidade. Localidades de grande porte tendem a receber para tratamento pacientes provenientes de pequenos municípios/microrregiões que possuem pouca ou nenhuma infraestrutura para detecção precoce e tratamento do câncer de mama. Muitos destes acabam por fixar ali sua residência ${ }^{20}$.

A ausência de recursos diagnósticos próximos à área de residência é uma barreira na prevenção de doenças como o câncer. Observa-se que estes recursos acabam se concentrando nos grandes centros urbanos, em áreas desenvolvidas e de melhor acesso para a população residente, porém distante e de difícil acesso para residentes de áreas periféricas e de baixo nível socioeconômico ${ }^{3}$. No ano de 2011, as macrorregiões Norte e Nordeste de Minas Gerais apresentaram razões de mamografia menores quando comparadas às macrorregiões Sul, Oeste e Triângulo. Centro e Sudeste apresentaram valores intermediários ${ }^{21}$. No Brasil, a maioria dos serviços credenciados no SUS para tratamento do câncer de mama estão localizados nas regiões Sul e Sudeste ${ }^{3}$. Em 2013, o número de mamografias realizadas nessas regiões foi maior do que nas regiões Norte e Centro-Oeste ${ }^{22}$.

No Norte de Minas Gerais, a microrregião de Montes Claros/Bocaiúva é a única que possui assistência hospitalar e ambulatorial para atenção oncológica. Dessa maneira, grande número de pessoas com câncer desloca-se para essa microrregião em busca de tratamento, o que sobrecarrega os dois hospitais de referência, dificulta o acesso aos serviços e atrasa a marcação de exames, procedimentos, cirurgias e outros serviços de que a população necessita ${ }^{23}$. Isto pode explicar as taxas mais elevadas de mortalidade por CAM desta microrregião em relação às outras da macrorregião Norte.

Ainda em Montes Claros/Bocaiúva, no período de 2006 a 2009, foi observado que 47,6\% das mulheres com diagnóstico por câncer de mama foram classificadas com estágio III e IV, 
Tabela 3. Modelos múltiplos da Regressão

\begin{tabular}{|c|c|c|c|c|c|c|c|}
\hline \multirow{2}{*}{ Variável $(n=77)$} & \multirow{2}{*}{$\begin{array}{c}\text { Modelo } \\
\text { Múltiplo } \\
\text { Distal }\end{array}$} & \multicolumn{6}{|c|}{ Modelos Múltiplos para Variáveis Proximais } \\
\hline & & M1 & M2 & M3 & M4 & M5 & M6 \\
\hline \multicolumn{8}{|l|}{ IDH } \\
\hline coeficiente & 0,097 & & & & & & \\
\hline p-valor & 0,632 & & & & & & \\
\hline \multicolumn{8}{|l|}{ IVS } \\
\hline coeficiente & 0,025 & & & & & & \\
\hline p-valor & 0,521 & & & & & & \\
\hline \multicolumn{8}{|l|}{ Renda Média per capita } \\
\hline coeficiente & 0,001 & & & & & & \\
\hline p-valor & 0,781 & & & & & & \\
\hline \multicolumn{8}{|l|}{ Grau de Urbanização (\%) } \\
\hline coeficiente & 0,125 & 0,133 & 0,124 & 0,137 & 0,131 & 0,134 & 0,126 \\
\hline p-valor & 0,006 & $<0,001$ & $<0,001$ & $<0,001$ & $<0,001$ & $<0,001$ & $<0,001$ \\
\hline \multicolumn{8}{|l|}{ População Feminina (log) } \\
\hline coeficiente & 0,595 & 0,779 & 0,616 & 0,761 & 0,726 & 0,764 & 0,697 \\
\hline p-valor & 0,205 & 0,070 & 0,159 & 0,058 & 0,070 & 0,062 & 0,079 \\
\hline \multicolumn{8}{|l|}{ Gastos per capita em saúde ( $R \$)$} \\
\hline coeficiente & & $-0,001$ & & & & & \\
\hline p-valor & & 0,745 & & & & & \\
\hline \multicolumn{8}{|l|}{ Razão de Médicos (por 1.000 hab.) } \\
\hline coeficiente & & & 0,353 & & & & \\
\hline p-valor & & & 0,568 & & & & \\
\hline \multicolumn{8}{|c|}{ Número de Mamógrafos (por 100.000 hab.) } \\
\hline coeficiente & & & & $-0,171$ & & & \\
\hline p-valor & & & & 0,562 & & & \\
\hline \multicolumn{8}{|l|}{ Cobertura ESF (\%) } \\
\hline coeficiente & & & & & $<0,001$ & & \\
\hline p-valor & & & & & 0,990 & & \\
\hline \multicolumn{8}{|c|}{ Distância percorrida para realização de mamografia (Km) } \\
\hline coeficiente & & & & & & 0,002 & \\
\hline p-valor & & & & & & 0,705 & \\
\hline \multicolumn{8}{|l|}{ Razão de Mamografia } \\
\hline coeficiente & & & & & & & 1,602 \\
\hline p-valor & & & & & & & 0,472 \\
\hline$R^{2}$ ajustado & 0,427 & 0,439 & 0,441 & 0,441 & 0,438 & 0,439 & 0,442 \\
\hline \multicolumn{8}{|l|}{ Resíduos do Modelo } \\
\hline Teste de Normalidade & 0,810 & 0,778 & 0,747 & 0,818 & 0,768 & 0,782 & 0,677 \\
\hline \multicolumn{8}{|l|}{ Jarque-Bera ( $p$-valor) } \\
\hline Teste de Heterocedasticidade & 0,081 & 0,060 & 0,066 & 0,028 & 0,061 & 0,051 & 0,052 \\
\hline \multicolumn{8}{|l|}{ Breusch Pagan ( $p$-valor) } \\
\hline Dependência espacial & 0,068 & 0,138 & 0,110 & 0,104 & 0,128 & 0,093 & 0,160 \\
\hline Moran's I ( $p$-valor) & & & & & & & \\
\hline
\end{tabular}

M1: Grau de Urbanização, População Feminina e Gastos Per Capita em Saúde; M2: Grau de Urbanização, População Feminina e Razão de Médicos; M3: Grau de Urbanização, População Feminina e Número de Mamógrafos; M4: Grau de Urbanização, População Feminina e Cobertura ESF; M5: Grau de Urbanização, População Feminina e Distância Percorrida para Realização de Mamografia; M6: Grau de Urbanização, População Feminina e Razão de Mamografia; N= 77 corresponde ao número de microrregiões de saúde de Minas Gerais 
sendo o percentual mais elevado no serviço público comparado ao privado. Além disso, 42,7\% tiveram o intervalo de tempo superior a 6 meses entre a suspeita clínica e a confirmação diagnóstica ${ }^{24}$. A falta de conhecimento em relação à doença e a escassez de recursos para assistência à saúde dificultam o diagnóstico e tratamento precoce do câncer de mama no Norte de Minas Gerais ${ }^{23}$.

A variável grau de urbanização explica $42,7 \%$ da variação das taxas de mortalidade das microrregiões de MG. Microrregiões com maior grau de urbanização, maior renda e alto índice de desenvolvimento humano estão localizadas, em sua maioria, nas macrorregiões do Triângulo Norte e Sul, Centro, Sul e Sudeste e são aquelas que apresentam as mais altas mortalidades por câncer de mama no estado.

Em Taiwan, no período de 1971 a 2008, o processo de urbanização foi associado à menor mortalidade padronizada por todas as causas, exceto para o câncer de mama ${ }^{25}$. No Brasil, estudo observou que nos estados e capitais mais urbanizados a taxa média de mortalidade por câncer de mama é maior quando comparada às dos menos urbanizado ${ }^{26}$. Revisão sistemática de literatura demonstrou que grau de urbanização de uma determina região está associado à maior incidência do câncer de mama ${ }^{27}$.

O processo de urbanização está associado a mudanças do estilo de vida como hábitos alimentares inadequados, redução da prática de atividade física, redução da caminhada para atividades diárias de vida devido ao uso de transporte público ou particular, gestação tardia ou inexistente, menor número de filhos e não amamentação. Esses fatores ou seu conjunto contribuem para o aumento da incidência do câncer de mama ${ }^{28,29}$.

Avaliando a sociedade e suas transformações, verifica-se que o estilo de vida das mulheres modificou-se ao longo dos anos, influenciando seus hábitos de vida e comportamentos. No que se refere aos fatores de risco reprodutivos, a maioria das mulheres residentes em regiões mais urbanizadas tende a adiar a maternidade utilizando anticoncepcionais. O seu uso após o quinto ano aumenta consideravelmente o risco para o CAM. Já a lactação exerce efeito protetor $^{30}$.

Estudo realizado no Serviço de Mastologia do Hospital das Clínicas da UFMG (Belo Horizonte, MG), com mulheres com diagnóstico recente de câncer de mama, constatou que $64,5 \%$ das pacientes utilizaram contraceptivo oral, $69 \%$ eram sedentárias, $58 \%$ apresentavam excesso de peso corporal, $93,5 \%$ excesso de gordura corporal e padrão dietético de má qualidade ${ }^{31}$.

As taxas de mortalidade, assim como as variáveis tanto do bloco socioeconômico quanto do assistencial apresentaram dependência espacial, ou seja, as microrregiões vizinhas apresentaram valores semelhantes entre si. Isto aponta para a clara divisão do território entre regiões de alta vulnerabilidade/baixo grau de urbanização, em direção ao Norte, e baixa vulnerabilidade/maior grau de urbanização, em direção ao Sul, visualizada quando traçada uma linha divisória no limite inferior das microrregiões de Unaí, João Pinheiro, Pirapora, Curvelo, Diamantina, Guanhães, Santa Maria do Suaçuí/São João Evangelista, Teófilo Otoni, Malacacheta/Itambacuri e Mantena.

A diversidade entre regiões pobres e ricas e a concentração entre aquelas que são mais parecidas são explicadas por diversos fatores, dentre eles, o papel de controle e comando de determinada região onde se concentram centros de gestão pública e empresarial ${ }^{32}$. Consequentemente essa região tem forte poder de influência sobre as que estão ao seu redor e articulam-se entre si. Quanto mais afastada uma área for dessa região central, menos semelhança ela terá.

Como limitação deste estudo, considera-se a obtenção de dados em bases secundárias, pois estas podem estar incompletas ou com erros de informação. Por exemplo, pode ter havido sub-registro de óbitos no período estudado, especialmente nas microrregiões mais pobres do estado. No entanto, estudos recentes têm mostrado melhora na qualidade das informações do $\mathrm{SIM}^{33,34}$. Uma outra limitação está relacionada ao desenho do estudo, pois, como os dados analisados são agregados por populações, todas as inferências feitas são para o nível ecológico, não havendo inferência direta para o nível individual, sob pena de incorrer na falácia ecológica. 
Este estudo demonstrou desigualdades regionais importantes, com alta mortalidade por câncer de mama nas regiões mais urbanizadas e populosas do estado, o que pode estar relacionado principalmente aos fatores de risco reprodutivos e hábitos de vida. Além disso, as áreas mais desenvolvidas possuem mais recursos para detecção precoce e tratamento do câncer de mama, contudo podem ocorrer diferenças na distribuição desses recursos dentro de um mesmo território, o que prejudicaria o acesso das mulheres residentes em áreas desprovidas de assistência.

A estruturação e planejamento dos municípios para o crescimento urbano pode contribuir com a definição de prioridades na alocação de recursos sociais, de saúde, materiais e humanos com mão de obra especializada e qualificada, diminuindo assim as diferenças e iniquidades entre as cidades.

As transformações da sociedade no decorrer dos anos influenciam as formas de adoecer e morrer das pessoas. É, portanto, relevante compreender as desigualdades entre as microrregiões de saúde e estabelecer estratégias para reduzi-las a partir do monitoramento geográfico das mudanças. Estas informações podem auxiliar na construção de uma rede assistencial igualitária, regionalizada e hierarquizada, a fim de reduzir desigualdades, investir melhor os recursos e prestar assistência de qualidade que atenda a mulher de forma integral desde a prevenção até o tratamento e/ou reabilitação.

\section{REFERÊNCIAS}

1. Bray F, Ferlay J, Soerjomataram I, Siegel RL, Torre LA, Jemal A. Global cancer statistics 2018: GLOBOCAN estimates of incidence and mortality Worldwide for 36 Cancers in 185 countries. CA Cancer J Clin. 2018;68(6):394-424. http://dx.doi.org/10.3322/caac.21492. PMid:30207593.

2. Siegel RL, Jemal A, Wender RC, Gansler T, Ma J, Brawley OW. An assessment of progress in cancer control. CA Cancer J Clin. 2018;68(5):329-39. http://dx.doi.org/10.3322/caac.21460. PMid:30191964.

3. Azevedo e Silva G, Bustamante-Teixeira MT, Aquino EM, Tomazelli JG, Dos-Santos-Silva I. Acesso à detecção precoce do câncer de mama no Sistema Único de Saúde: uma análise a partir dos dados do Sistema de Informações em Saúde. Cad Saude Publica. 2014;30(7):1537-50. http://dx.doi.org/10.1590/0102311X00156513. PMid:25166949.

4. Fitzmaurice C, Akinyemiju TF, Al Lami FH, Alam T, Alizadeh-Navaei R, Allen C, et al. Global, regional, and national cancer incidence, mortality, years of life lost, years lived with disability, and disability-adjusted life-years for 29 Cancer Groups, 1990 to 2016: a Systematic Analysis for the Global Burden of Disease Study. JAMA Oncol. 2018;4(11):1553-68. http://dx.doi.org/10.1001/jamaoncol.2018.2706. PMid:29860482.

5. Girianelli VR, Gamarra CJ, Azevedo e Silva G. Os grandes contrastes na mortalidade por câncer do colo uterino e de mama no Brasil. Rev Saude Publica. 2014 jun;48(3):459-67. http://dx.doi.org/10.1590/S00348910.2014048005214. PMid:25119941.

6. Instituto Nacional do Câncer. Conceito e magnitude do câncer de mama [Internet]. Rio de Janeiro: INCA; 2019 [citado em 2019 set 10]. Disponível em: https://www.inca.gov.br/controle-do-cancer-de-mama/ conceito-e-magnitude

7. Medeiros M, de Souza PH, de Castro FÁ. A estabilidade da desigualdade de renda no Brasil: 2006-2012: estimativa com dados do imposto de renda e pesquisas domiciliares. Cien Saude Colet. 2015 abr;20(4):97186. http://dx.doi.org/10.1590/1413-81232015204.00362014. PMid:25923610.

8. Instituto Nacional de Câncer José Alencar Gomes da Silva. A situação do câncer de mama no Brasil: síntese de dados dos sistemas de informação. Rio de Janeiro: INCA; 2019.

9. Instituto Brasileiro de Geografia e Estatística. Minas Gerais. Sinopse do Censo Demográfico 2010 [Internet]. Rio de Janeiro: IBGE; 2016 [acesso em 20 jan 2016]. Disponível em: http://www.ibge.gov.br/estadosat/ temas.php?sigla=mg\&tema=sinopse_censodemog2010

10. Brasil. Governo Federal. Acesso à informação [Internet]. 2016 [acesso em 20 jan 2016]. Disponível em: http://www.acessoinformacao.gov.br

11. Doll R, Payne P. Waterhouse. Cancer incidence in five continents: a technical report. Berlin: Springer-Verlag; 1966. http://dx.doi.org/10.1007/978-3-642-85849-9.

12. Organização Pan-Americana de Saúde. Rede Interagencial de Informação para a Saúde. Indicadores básicos para a saúde no Brasil: conceitos e aplicações. 2. ed. Brasília: Organização Pan-Americana da Saúde; 2008.

13. Pitchon A, Girodo AM, Gomes CC, Gomes DHP, Júnior FGP, Freire F, et al. Índice de Vulnerabilidade Social (IVS) 2012. Belo Horizonte: Prefeitura Municipal de Belo Horizonte; 2013. 
14. Victora CG, Huttly SR, Fuchs SC, Olinto MT. The role of cocneptual frameworks in epidemiological analysis: a hierarchical approach. Int J Epidemiol. 1997;26(1):224-7. http://dx.doi.org/10.1093/ije/26.1.224. PMid:9126524.

15. Figueiredo FWS, Adami F. Effects of the high-inequality of income on the breast cancer mortality in Brazil. Sci Rep. 2019;9(1):8-13. http://dx.doi.org/10.1038/s41598-019-41012-8.

16. Di Sibio A, Abriata G, Forman D, Sierra MS. Female breast câncer in Central and South America. Cancer Epidemiol. 2016;44(Suppl 1):S110-20. http://dx.doi.org/10.1016/j.canep.2016.08.010. PMid:27678313.

17. Sistema de Informação sobre Mortalidade - SIM. Banco de dados do Sistema Único de Saúde - DATASUS. Serviços. Transferência/Dowload de Arquivos/Arquivos de Dados [Internet]. 2019 [acesso em 12 jul 2019]. Disponível em: http://www2.datasus.gov.br/DATASUS/index.php?area=0901.

18. Programa de Avaliação do Desempenho do Sistema de Saúde - PROADESS. Matriz de Indicadores. Regiões de Saúde [Internet]. 2019 [acesso em 12 jul 2019]. Disponível em: https://www.proadess.icict.fiocruz.br/ index.php?pag=matraba.

19. Cardoso DF, Ribeiro LCS. Índice relativo de qualidade de vida para os municípios de Minas Gerais. Planej. Polít. Públicas. 2015;45:347-75.

20. Couto MAS, Guerra MR, Firme VAC, Bustamante-Teixeira MT. Comportamento da mortalidade por câncer de mama nos municípios brasileiros e fatores associados. Rev Panam Salud Publica. 2017;41:1-10. http:// dx.doi.org/10.26633/RPSP.2017.168.

21. Corrêa CSL, Pereira LC, Leite ICG, Fayer VA, Guerra MR, Bustamante-Teixeira MT, et al. Rastreamento do câncer de mama em Minas Gerais: avaliação a partir de dados dos sistemas de informações do Sistema Único de Saúde. Epidemiol Serv Saude. 2017;26(3):481-92. http://dx.doi.org/10.5123/S167949742017000300006. PMid:28977173.

22. Assis M, Ramos DN, Tomazelli JG. Detecção precoce do câncer de mama no brasil: um olhar a partir dos exames realizados no SUS [Internet]. Rio de Janeiro: INCA; 2014 [acesso em 20 jan 2016]. Disponível em: http://saudepublica.bvs.br/pesquisa/resource/pt/sus-30348

23. Alves MO, Magalhães SCM, Coelho BA. A regionalização da saúde e a assistência aos usuários com câncer de mama. Saúde Soc São Paulo. 2017;26(1):141-54. http://dx.doi.org/10.1590/s0104-12902017160663.

24. Soares PB, Quirino S Fo, de Souza WP, Gonçalves RC, Martelli DR, Silveira MF, et al. Características das mulheres com câncer de mama assistidas em serviços de referência do Norte de Minas Gerais. Rev Bras Epidemiol. 2012 set;;15(3):595-604. http://dx.doi.org/10.1590/S1415-790X2012000300013. PMid:23090306.

25. Chen BK, Yang CY. Differences in age-standardized mortality rates for avoidable deaths based on urbanization levels in Taiwan, 1971-2008. Int J Environ Res Public Health. 2014;11(2):1776-93. http:// dx.doi.org/10.3390/ijerph110201776. PMid:24503974.

26. Ribeiro MS, Abreu NC, Borges TFF, Guimaraes RM, Muzi CD. Urbanidade e mortalidade por cânceres selecionados em capitais brasileiras, 1980-2009. Cad. Saúde Colet. 2013;21(1):25-33. https://doi. org/10.1590/S1414-462X2013000100005.

27. AkinyemijuTF, Genkinger JM, Farhat M, Wilson A, Gary-Webb TL, Tehranifar P. Residential environment and breast cancer incidence and mortality: a systematic review and meta-analysis. BMC Cancer. 2015;15:191. http://dx.doi.org/10.1186/s12885-015-1098-z. PMid:25885593.

28. Bray F, Piñeros M. Padrões, tendências e projeções do câncer na América Latina e no Caribe: um contexto global. Salud Publica Mex. 2016 abr;58(2):104-17. http://dx.doi.org/10.21149/spm.v58i2.7779. PMid:27557369.

29. Wen D, Wen X, Yang Y, Chen Y, Wei L, He Y, et al. Urban rural disparity in female breast cancer incidence rate in China and the increasing trend in parallel with socioeconomic development and urbanization in a rural setting. Thorac Cancer. 2018;9(2):262-72. http://dx.doi.org/10.1111/1759-7714.12575. PMid:29280294.

30. Lauter DS, Berlezi EM, Rosanelli CLSP, Loro MM, Kolankiewicz ACB. Câncer de mama: estudo caso controle no Sul do Brasil. Rev Cienc Salud. 2014;7(1):19-26.

31. Oliveira DR, Carvalho ES, Campos LC, Leal JA, Sampaio EV, Cassali GD. Avaliação nutricional de pacientes com câncer de mama atendidas no Serviço de Mastologia do Hospital das Clínicas, Belo Horizonte (MG), Brasil. Cien Saúde Coletiva. 2014;19(5):1573-80. http://dx.doi.org/10.1590/1413-81232014195.02262013. PMid:24897222.

32. Pinheiro OM. Plano diretor e gestão urbana. 3. ed. Brasília: CAPES, UAB; 2014.

33. Lino RRG, Fonseca SC, Kale PL, Flores PVG, Pinheiro RS, Coeli CM. Tendência da incompletude das estatísticas vitais no período neonatal, estado do Rio de Janeiro, 1999-2014. Epidemiol Serv Saude. 2019;28(2):e2018131. http://dx.doi.org/10.5123/S1679-49742019000200014. PMid:31291437. 
34. Queiroz BL, Freire FHMA, Gonzaga MR, Lima EEC. Estimativas do grau de cobertura e da mortalidade adulta (45q15) para as unidades da federação no Brasil entre 1980 e 2010. Rev Bras Epidemiol. 2017;20(Supl 01):21-33. http://dx.doi.org/10.1590/1980-5497201700050003. PMid:28658370. 